dustrie dans ces régions. Du côté des producleurs, ce même effort a permis d'utiliser au maximum les ressources hydrauliques de ces régions. L'expérience a démontré que la voie suivie étäit la bonne. Il est souhaitable d'appliquer ces formules à des zones plus étendues, mais en onérant avec prudence et progressivement, en constituant d'abord des groupements régionaux, puis ensuile des supergroupements. On arrivera ainsi petit à petit à faciliter la répartition des énergies sur l'ensemble de notre territoire.

Quant aux connexions internationales, pour l'instant lout au moins, la réalisation d'un tel projet présenterait des difficultés techniques sérieuses, tenant notamment à la nécessité d'employer des tensions au moins égales à $400 \mathrm{kV}$.; elle exigerait des capitaux dont la réunion serait malaisée et la rémunération très douteuse.

Restant à l'U. P. E. P. O. et ses dépendances, on constate que la conception qui a présidé à leur création était saine et féconde et que grâce aux efforts conjugués de l'Administration et des Sociétés privées, les résultats obtenus ont réponduaux espérances du début.

\title{
DOCUMENTATION
}

\section{Les installations électriques automatiques de la ligne d'Orléans à Tours, du réseau de la Compagnie de chemin de fer de Paris à Orléans}

\section{I.- Introduction}

Dans une étude fort documentée publiée par la " Revue Générale de l'Electricité " du 14 juillet 1934, M. Mores a exposé d'une manière très complète, et en même temps fort judicieuse, les - méthodes employées par la Cie des Chemins de fer de Paris à Orléans, pour équiper sous forme automatique les sous-stations du personnel de manœuyre des sous-stations, réduction d'autant plus désirable que des difficultés d'habitat se posaient pour ces postes toujours très isolés, aurait suffi comme raison déterminante à l'adoption intégrale de l'automatisme.

C'est l'étude de M. Morel que nous résumons ci-après, assez largement du reste, en raison de l'importance exceptionnelle posée par ce problème de l'automatisme.

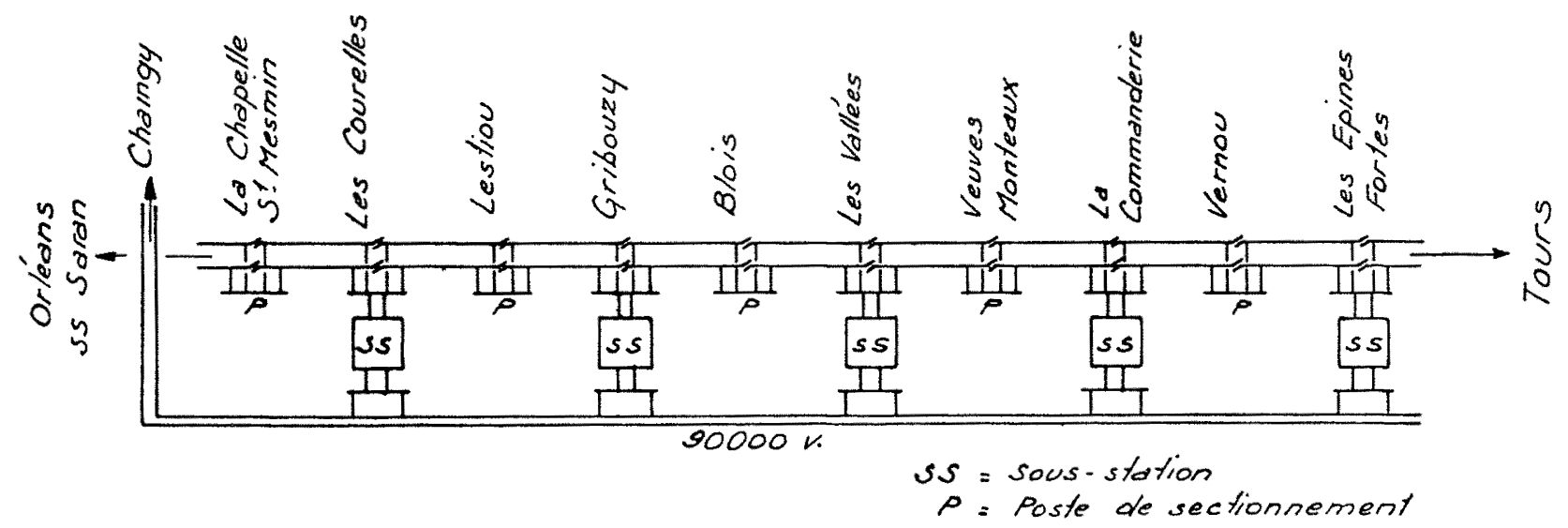

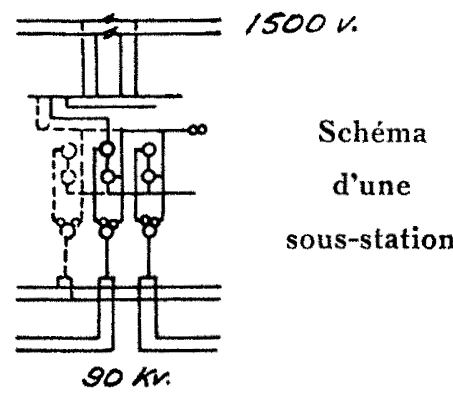

de traction du secteur d'Orléans à Tours, desservi par cette Compagnie.

On sait que celle-ci, après avoir sensiblement modifié ses plans primitifs d'electrification, notamment après avoir abandonnć provisoirement l'électrification de certaines lignes de montagne trop peu payantes du Massif Central, s'est assigné l'èlectrification de Paris à Orléans-Vierzon avec prolongement ultérieur jusqu’à Brive, et d'Orléans-Tours, cette dernière opération ayant été tout récemment achevée au prix detravaux poussés très rapidement et en même temps sous une forme véritablement intéressante en tant yu'adoption nouvelle de l'automatisme. La réduction
Fig. 1. - Schéma des sous-stations de traction de la ligne d'Orléans à Tours.

\section{II. - Schéma de l'installation}

Ainsi, les essais entrepris de divers côtés et, en particulier, à la sous-station de Tivernon sur la ligne de Paris à Vierzon, ont ćté assez concluants pour généraliser l'emploi de la commande automatique dans les nouvelles installations. C'est ainsi que les cinc sous-stations de la nouvelle ligne d'Orléans à Tours furent dotées de la commande automatique. Cette nouvelle ligne a été inaugurce au mois de juillet de l'année 1933 (1).

La ligne en question s'étend des Aubrais à Tours sur une longucur de $118 \mathrm{~km}$. Elle est à double voie et assure un trafic très important. Les deux lignes caténaires sont alimentées par cinq sous-stations indiquées sur la fig. 1. Chaque sous-station est sćparće de la sous-station voisine par un poste de sectionnement.

(1) Voir pour plus de détails, comme indiqué ci-dessus, l'article de M. Morel, dans la Revue ${ }_{2}$ Générale de l'Eleclricilé du 14 Juillet 1934, p. 61-77, 14 fig. 
Les lignes de contact sont sectionnées au droit de chaque poste ou sous-station, et les tronçons mis en parallèle par la barre générale du poste ou de la sous-station.

Chaque sous-station est équipéc de deux groupes transformateur-commutatrice, chacin de $2000 \mathrm{kWV}$. sous 1500 volts. L'installation d'un troisième groupe est prévue. Chaque groupe comporte deux commutatrices d'excitation hypercompound en série, de $1000 \mathrm{~kW}$. chacune sous 750 volts.

L'alimentation en haute tension est assurée par le poste de transformation de Chaingy, au moyen de deux lignes triphasées de $90 \mathrm{kV}$. en parallèle.

\section{III. - Principe de la commande automatique des sous-stations}

Le fonctionnement des sous-stations est entièrement automatique et, sauf les disjoncteurs de feeders, toute commande manuelle de secours est supprimée. Normalement les disjoncteurs de feeders sont fermés et assurent la continuité de la ligne. Celle-ci est maintenue constamment à la tension de 1500 volts par les deux sousstations de Saran et des Epines-Fortes, appelées sous-stations " pilotes " et qui marchent sans, arrêt.

Considérons dans ces conditions les deux sources $S$ et $S$ 'à la même tension (fig.2) et quatre sous-stations $\mathrm{A}, \mathrm{B}, \mathrm{C}, \mathrm{D}$, que nous

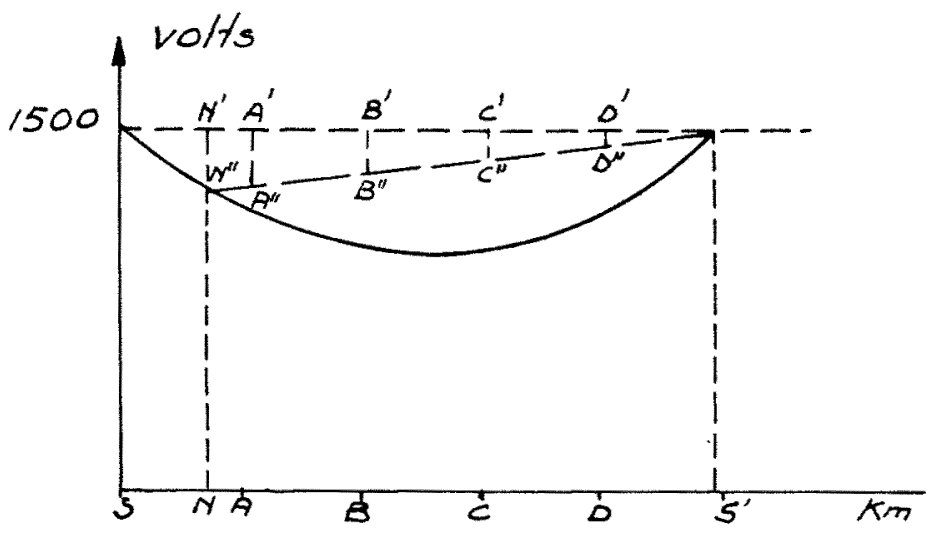

Fig. 2. - Courbe des chutes de tension le long d'une ligne de contact homogène.

supposons à l'arrêt. Un train partant de $\mathrm{S}$, s'avançant vers $\mathrm{A}$, crée une chute de tension, qui se représente par une parabole, si l'intensité du courant absorbé par la locomotive est constante. Le démarrage de la sous-station est basé sur cette chute de tension qui est décelée au moyen d'un relais voltmétrique sensible. L'arrêt de la sous-station est provoqué par un relais ampèremétrique dès que l'intensité tombe au dessous d'une certaine valeur. Ces deux relais sont temporisés afin d'éliminer les baisses de tension fugitives, ainsi que les manques de charge de courte durée.

Ce système de démarrage par baisse de tension nécessitait deux précautions spéciales :

$1^{0}$ Il fallait éviter ia surélévation brusque de tension au moment du couplage; on a simplement profité à cet effet de la grande réactance des transformateurs, nécessaire pour réaliser la caractéristique hypercompound des commutatrices.

$2^{\circ}$ Avec les trains rapides actuels, la chnte de tension croît très vite; pour éviter des chutes de tension excessives sur la locomotive, il est d'une importance capitale d'accélérer le démarrage des groupes. On est arrivé à des résultats satisfaisants en démarrant à demi-tension en moteur asynchrone, grâce à une importance cage d'écureuil. Le groupe est prêt à être couplé au bout de 30 secondes.

\section{IV. - Fonctionnement automatique d'un groupe de traction à commutatrices}

En dehors de la rapidité des opérations de démarrage, une sécurité complète est indispensabje. Ces conditions sont réalisées par l'utilisation d'un appareillage robuste et approprié.

Le démarrage complet du groupe comporte les phases suivantes :

10 Fermeture d'un contacteur général de contrôle, qui agit sur tous les circuits de commande; son ouverture provoque la remise au repos immédiate de l'appareillage et l'arrêt du groupe;

20 Fermeture de l'interrupteur dans l'huile à $90000 \mathrm{~V}$.;

30 lómarrage simultané des deux commutatrices:

10 Polarisation, autoexcitation, passage à la pleine tension;

$5^{\circ}$ Abaissement des halais du côté terre;

$6^{\circ}$ Réglage de la tension du côté ligne à nne valeur supéricure de 1 pour 100 sur la tension du réseau;

$7^{\circ}$ Abaissement des balais de la commutatrice du côté de la ligne ;

So Couplage du groupe.

On trouve, par machine, un relais de synchronisme, un relais de polarisation et un relais d'autoexcitation. La fermeture successive de ces relais est indispensable pour que s'effectue la suite des opérations de démarrage. Comme nous venons de le dire, le couplage est précédé par le réglage de la tension du groupe. Cette opération s'effectue par le réglage des rhéostats d'excitation, commandés par des moteurs, contrôlés à leur tour par des relais voltmétriques du type balance.

En cas de défaillance des sous-stations pilotes ou d'isolement d'un poste de sectionnement, toutes les sous-stations peuvent être pilotes.

Le second groupe démarre par avarie ou surcharge du premier et s'arrête si la charge ne justifie pas sa marche. Le troisième groupe, quand il sera installé, jouera un rôle analogue vis-à-vis des deux premiers. Comme le premier groupe a un service beaucoup plus dur que les suivants, il est nécessaire de changer périodiquement l'ordre de priorité des groupes, ce qui se fait à l'aide d'un commutateur manuel. Les mises en route des groupes second et troisième sont commandées par des relais de surcharge ou des relais d'avarie. L'indication de démarrage n'est pas supprimée sur le groupe avarié, qui pourra être remis en service dès que l'avarie sera résolue. Le groupe qui a pris sa place será éliminé après son couplage sur le réseau. En général l'arrêt d'un groupe s'obtient par suppression du courant sur le contacteur général de contrôle, déjà mentionné.

Nous avons déjà indiqué que les relais de baisse de tension et de surcharge sont temporisés. Il en est de même du relais d'avarie, mais la temporisation de celle-ci est très courte : de l'ordre de 3 secondes. D'ailleurs, aussi bien les valeurs d'ouverture ou de fermeture de tout relais, que leur temporisation, sont indépendamment réglables, ce qui donne au système une grande souplesse, lui permettant de s'adapter facilement aux exigences du trafic

\section{V.- Protection des groupes}

On distingue les appareils de protection à arrêt permanent du groupe, de ceux qui ne produisent qu'un arrêt temporaire, la perturbation étant susceptible de disparaître d'elle même.

Les premiers, presque tous à réarmement manuel, provoquent l'ouverture dı contacteur général de commande et l'empêchent de se refermer. Ce sont : un relais de maximum de courant alternatif par groupe, un relais d'échauffement par palier, un relais d'amorçage d'arc au collecteur ou de mise à la masse par commutatrice, un relai d'échauffement de transformateur et un relais de commande de l'équipement automatique. Ce dernier mesure simplement le temps de démarrage : si les opérations de démarrage ne sont pas terminées en un temps donné, le relais arrête le groupe jusqu'à inspection.

Les appareils à arrêt temporaire comprennent : un relais de vérification de la tension de la batterie, les disjoncteurs ultrarapides du groupe, un relais d'échauffement des enroulcments par commutatrice, un relais à inversion de polarité par groupe et un relais de retour de courant par groupe.

Le relais de vérification de la tension de la batterie se justifie par l'importance des appareils branchés sur son circuit : l'interrupteur à huile et les disjoncteurs rapides. Le relais à retour de courant sert à mettre la sous-station à l'arrêt au cas où, par suite d'un déclenchement sur la ligne à $90000 \mathrm{~V}$. elle viendrait à fonctionner à sens de courant inverse, transformant le courant continu en alternatif. On évite ainsi le couplage de deux réseaux non en phase, par réenclenchement intempestif des disjoncteurs à haute tension. 


\section{VI. - Départs de feeders et postes de sectionnement}

Les disjoncteurs alimentant les lignes de contact constituent la protection éloignée des groupes. En cas de court-circuit en ligne, afin de limiter les perturbations sur le trafic, ils auront pour but d'isoler le tronçon défectueux à l'exclusion de tout autre.

Le disjoncteur ultra-rapide des départs et des postes de sectionnement (1) présente la particularité suivante : le déclenchement s'opère par une spire parcourue par une partie du courant principal. Cette spire est shuntée par une barre inductive, de telle sorte qu'en cas de variation brusque de courant, celui-ci se répartissant entre le shunt et la spire, suivant les impédances et non suivant les résistances, le point de déclenchement statique s'en trouve notablement abiassé. Ce disjoncteur est donc sensible aux accélérations de courant. De plus, il est polarisé : le flux de la spire précédente doit être en opposition avec celui de la bobine de maintien. Ces deux propriétés rendent ce disjoncteur spécialement intéressant pour la sélection des défauts.

\section{VII. - Sélection des défauts}

Considérons les sous-stations $S$ et $S^{\prime}$ séparées par le poste de sectionnement $P$ (fig. 3). Chaque disjoncteur de sous-station est lié par un fil pilote au disjoncteur correspondant de la sous-station voisine; tout déclenchement de $\mathrm{A}$ entraîne celui de $\mathrm{A}^{\prime}$, de $\mathrm{G}$
Grâce au fil pilote, la position des disjoncteurs de poste est contrôlée à chaque instant par les sous-stations. Ceci est important en raison de la situation des postes laissés sans surveillance, loin de tout personnel qualifié. Cette même raison a conduit à compléter la commande automatique des disjoncteurs de départ par une commande manuelle.

\section{IX.- Dispositions accessoires et exploitation}

$1^{\circ}$ Manque de tension alternative. - Si le manque de tension alternative n'intéresse que la ligne d'Orléans à Tours (déclenchement à Chaingy), les caténaires restent alimentés par la sousstation pilote de Saran. Les. autres sous-stations déclenchent, comme nous l'avons déjà dit, par retour de courant. Mais la continuité de la ligne caténaire subsiste en général et, dès le rétablissement de la tension alternative, les sous-stations intéressées se remettent automatiquement en service.

Par contre, dans le cas d'un manque de tension générale sur la ligne à haute tension, les caténaires ne sont plus alimentés et tous les disjoncteurs de feeders déclenchent, faute de tension aux bornes de leurs bobines de maintien, qui sont justement alimentées, comme nous l'avons dit, par le caténaire. Dans ce cas, grâce à des relais de baisse de tension, dès le retour de la tension alternative, les sous-stations pilotes démarrent, immédiatement suivies

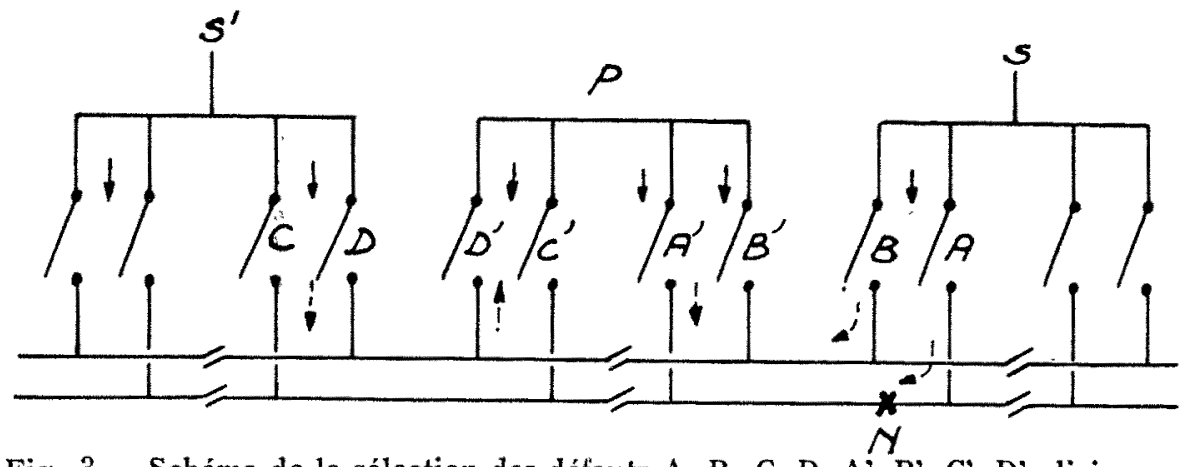

Fig. 3.- Schéma de la sélection des défauts A, B, C, D, A', B', C', D', disjoncteurs ; $\mathrm{N}$ défaut, $\mathrm{P}$ poste de sectionnement, $\mathrm{S} S$ ' sous-stations. Les flèches en trait plein indiquent le sens de déclenchement, celles en trait ponctué le sens du courant de défaut.

celui de C', etc. Supposons une mise à la terre au point $N$. Le défaut est alimenté par $S$, directement à travers $A$ et indirectement à travers $\mathrm{B}, \mathrm{B}^{\prime}, \mathrm{A}^{\prime}$; de même il est alimenté par $\mathrm{S}^{\prime}$ à travers $C, C^{\prime}, D, D^{\prime}$ et $A^{\prime}$. Le disjoncteur $A^{\prime}$ sera parcouru parla somme des courants traversant $B^{\prime}, C^{\prime}, D^{\prime}$. De plus, les bobines de maintien des disjoncteurs étant alimentées par la caténaire, celle de A' verra la tension baisser à ses bornes, donc s'affaiblir son action. A' aura ainsi toutes chances à déclencher et il fera déclencher $\mathrm{A}$ par le fil pilote. De toute façon, si' un défaut se produit, l'un ou l'autre des disjoncteurs limitant le tronçon intéressé déclenchera avant les autres et fera déclencher par le fil pilote le disjoncteur à l'autre extrémité, si l'intensité du courant à travers la barre de ce dernier n'a pas atteint une valeur suffisante. Des oscillogrammes enregistrés au cours d'essais en court-circuit démontrent la nécessité du fil pilote, sans quoi, l'un des disjoncteurs limitant le départ pourrait ne pas déclencher et continuer à alimenter le défaut, pour peu que celui-ci soit un peu éloigné.

\section{VIII. - Essais de la ligne et réenclenchement automatiques}

Dès que le tronçon défectueux est isolé, un apparëillage approprié se met en activité pour l'essayer. L'essai s'effectue en alimentant le défaut par la barre générale positive de la sous-station, à travers une résistance de $40 \mathrm{ohms}$. Un relais balance compare la tension aux bornes de la résistance à la tension de la barre générale positive. Si le défaut subsiste, on fait trois essais successifs séparés par deux intervalles de repos; si le troisième essai est négatif, le disjoncteur de départ est bloqué et l'avarie signalće. Si le défaut a disparu, la tension aux bornes de la résistance est plus faible, le relais ferme son contact et provoque le réenclenchement du disjoncteur de sous-station, qui est suivi du réenclenchement du disjoncteur de poste.

(1) Voir l'article de MM, Léon Vernier et Maurice Wilfart : "Le disjoncteur extra-rapide de Thomson-Houston "), R.G.E., 30 aout et 6 septembre 1924 , t. XYI, p. $339-351$ et $387-397$. de chacune des quatre autres, dans un ordre fixé par des relais de temporisation.

$2^{\circ}$ Sources auxiliaires pour les circuits de commande. - On utilise pour la conmande automatique soit du courant alternatif à 21.5 volts, soit du courant continu à 125 volts, fourni par une batterie d'accumulateurs. En général, l'alimentation par la batterie est réservée à la commande des interrupteurs dans l'huile et des disjoncteurs ultra-rapides, ainsi qu'à l'équipement automatique des feeders, ceux-ci devant rester autant que possible en service, même en cas de manque de tension alternative.

$3^{\circ}$ Rôle du personnel dans les sous-stations automatiques. - I.e personnel de chaque sous-station automatique est réduit à trois agents : un électricien et deux aides, au lieu des sept agents d'une sous-station manuelle. La présence de ce personnel est justifiée par les manœuvres sur les lignes à $90000 \mathrm{~V}$. ou sur les caténaires, et par les liaisons téléphoniques. En dehors de l'entretien, il n'a à intervenir dans le fonctionnement de l'équipement automatique, qu'en cas de dérangement. Une signalisation très complète et un schéma lumineux indiquent à l'agent, immédiatement, la perturbation survenue. L'appareillage automatique ne comporte d'ailleurs que des relais et contacteurs ayant chacun leur jonction et disposés sur des panneaux. L'agent aura toutes facilités pour remédier aux défaillances passagères, d'ailleurs rares, de l'équipement automatique.

\section{X. - Conclusion}

Cet équipement automatique installé par la société Als-Thom a donné toute satisfaction, et certaines craintes concernant son fonctionnement se sont trouvées sans fondement. Les sous-stations restent arrêtées quatre à cinq heures par jour et le rendement moyen est de l'ordre de 86 pour 100 . Ainsi que de nombreuses autres sous-stations analogues, ces nouvelles installations semblent encore démontrer la souplesse, l'économie ct la sécurité des équipemnts automatiques. 


\section{Les rames automotrices rapides Diesel électriques de la Compagnie du Nord ${ }^{(1)}$}

La Compagnie du Nord a mis en service, le 27 juillet 1931, deux rames automotrices destinées à assurer le service Paris-Lille. Elles parcourent cette distance $(258 \mathrm{~km}$.) tous les jours, à une vitesse commerciale de $90 \mathrm{~km} / \mathrm{h}$., environ, la vitesse maximum étant de $140 \mathrm{~km}$./h. Aux essais, on a atteint $160 \mathrm{~km} / \mathrm{h}$.

Une rame est composée de deux motrices accouplées entre lesquelles est intercalée une remorque. La forme extérieure a été déterminée à la suite d'essais effectués au tunnel aérodynamique. Ces essais ont mis en évidence que la résistance de l'air se trouve considérablement accrue :

$1^{0}$ Par les dénivellations habituelles : protubérance ou dépressions, des baies, des portes, des soufflets de cuir, etc.;

$2^{\circ}$ Par les tourbillons provoqués par le passage à grande vitesse d'appareils de formes très discontinues à faible distance du sol.

Par conséquent, dans la construction de la carrosserie, on a pris les précautions suivantes :

$1^{0}$ Les glaces des baies sont en retrait seulement de $4 \mathrm{~mm}$. par rapport à la surface extérieure des faces; le plan des portes coïncide avec le plan des faces; le soufflet de cuir habituel a été remplacé par une enveloppe de caoutchouc tendue entre deux caisses, de facon à assurer la continuité de la forme extérieure;

$2^{\circ}$ On a adopté sous le iongeron un tableau en tôle de duralumin, qui protège contre le vent toutes les formes plus ou moins discontinues (essieux, moteurs, etc.), sous la caisse.

Ces précautions ont permis de réduire de $60 \%$ le coefficient de résistance aérodynamique, par comparaison avec un véhicule de chemin de fer ordinaire.

La rame est entièrement symétrique de façon à pouvoir circuler indifféremment dans les deux sens.

Les motrices sont du type 2-B, avec un bogie générateur (supportant le groupe électrogène) et un bogie moteur. Le groupe électrogène repose sur un châsssis auxiliaire, suspendu au châssis principal en six points, par des silents blocs et des accouplements à rotules. Les essieux moteurs sont actionnés chacun par un moteur suspendu par le nez, avec interposition de ressorts de caoutchouc Spencer-Moulton.

Les moteurs Diesel sont à 12 cylindres, disposés en V, sous un angle de $60^{\circ}$. La puissance unihoraire est de $410 \mathrm{ch}$. et le poids seulement de $1950 \mathrm{~kg}$, grâce à la grande vitesse de rotation de $1400 \mathrm{t} / \mathrm{m}$. Les masses tournantes sont équilibrées ; d'autre part, un amortisseur spécial en bout d'arbre supprime les vibrations dues à l'élasticité du vilebrequin, de façon que l'on n'a pas à craindre de vitesse critique.

L’injection du combustible est effectuée par le procédé de pulvé- risation directe, qui offre l'avantage d'une chambre de combustion simple et d'une bonne combustion à toutes les charges. Chaque cylindre est pourvu d'une pompe d'injection. L'ensemble de ces pompes est commandé par l'intermédiaire d'un régulateur d'injection qui agit sur le débit de ces pompes. Ce régulateur se compose essentiellement d'un piston à pression d'huile sur une de ses faces et sous l'action d'un fort ressort sur sa face opposée. Le ressort commande le mouvement de fermeture, de façon que la fermeture est assurée, dans le cas où la pression d'huile viendrait à tomber au dessous d'une certaine limite, (car c'est cette même pression qui règne également dans le circuit de graissage

La tête du ressort est commandée par un régulateur tachymétrique, de façon à maintenir la vitesse à une valeur constante; le conducteur a simplement à fixer la valeur de cette vitesse.

Le démarrage est effectué au moyen de la génératrice principale, qui fonctionne alors en moteur, alimentée par la batterie de démarrage. On peut éventuellement démarrer à l'air comprimé.

La transmission électrique est du système Gebus. On sait (c'est le système le plus simple) qu'il consiste à employer une génératrice shunt à faible saturation, dont on prévoit les caractéristiques de fonctionnement, de façon que la puissance demandée au moteur Diesel reste, autant que possible, constante pour toute valeur de l'effort de traction, sans intervențion du mécanicien.

La dynamo ne s'amorce qu'à une vitesse d'environ $800 \mathrm{t} / \mathrm{m}$; le courant est alors progressivement envoyé dans les moteurs, d'où il résulte un démarrage très doux.

La génératrice comporte un enroulement série, utilisé pendant le démarrage du moteur Diesel, quand la génératrice fonctionne comme moteur série.

Le freinage est du système oléo-pneumatique Lockheed. Unc particularité intéressante de ce système constitue en son régulateur centrifuge qui règle automatiquement la pression sur les sabots en fonction de la vitesse du véhicule, pour tenir compte des variations du coefficient de frottement avec cette vitesse. Il existe de plus, pour le freinage d'urgence, un frein électromagnétique.

Le chauffage de la rame est à eau chaude, fournie par une chaudière à charbon.

I.e dispositif d' "homme mort" provoque l'arrêt du moteur Diesel et le fonctionnement des freins, si le conducteur lâche la poignée de conduite pendant plus de-six secondes. Une sonnerie retentit dès qu'il lâche la poignée.

$$
\text { J. B. }
$$

(1) D’après Le Génie Civil du 15 septembre lỳ34.

\section{Sur le freinage des automotrices de chemin de fer ${ }^{(1)}$}

L'emploi de plus en plus répandu d'automotrices de chemin de fer a posé un certain nombre de problèmes d'exploitation qui ne sont pas encore entièrement résolus, au moins sous forme d'un avis unanime.

Parmi ces questions, celle du freinage est la plus importante, car si l'on demande aux automotrices des vitesses fortement accélérées, il faut réclamer d'elles la même sécurité, toutes choses égales, en matière d'arrêt devant un obstacle imprévu.

C'est cette question du freinage qui est traitée dans le Bulletin de la Société des Ingénieurs Civils, mars-avril 1934, par M. BORDE, avec beaucoup de compétence. Il compare les avantages des freins à frottement agissant sur les roues avec ceux actionnant des tambours solidaires de ces mêmes roues. Il examine également la question des freins à frottement sur rails. En particulier, il étudie les freins à frottement sur roues suivant la nature des surfaces frottantes, le mode de régulation d'efforts de freinage, la transmission et l'amplification de l'effort initial de celui-ci.

Toute cette étude est à lire, car elle s'émaille de comparaisons inusitées. Citons les conclusions de l'auteur :

Conclusion. - On peut retenir certains caractères généraux concernant les freins d'automotrices, déjà expérimentées à l'époque actuelle, quil constitue toutefois encore une période d'essais et d'observations.

Freins à frottement sur roues. -- Tendance en faveur des freins à tambour, mais après mise au point de ceux-ci, quant à leur accessibilitć et facilité d'entretien.

Transmission des efforts de freinage aux surfaces de frottement, aussi simple et sûre que possible.

Commande du frein. - De préférence pneumatique, automatique ou directe, mais avec des précautions spéciales en vue de la sécurité, dans ce dernier cas.

La variation automatique de la pression pourra améliorer le fonctionnement du frein à sabots aux grandes vitesses.

Le frein extcrne du type électrumagnétique sur rails rendra des services comme frein de service ou comme frein d'urgence, suivant des conditions que l'expérience fixera.

L. B.

(1) M. Borde, Bulletin de la Sociélé des Ingénieurs Civils, Mars" Avril 1934 